\title{
Programa para ayudar a estudiantes de tercer año a integrar el contenido y la forma en la realización de la historia clínica
}

\author{
Roger Ruiz-Moral, Isabel Salido-Morales, Cristina Aguado-Taberné, Luis A. Pérula de Torres, \\ Antonia Alba-Dios, Francisco Guerra-Pasadas
}

Introducción. Las habilidades de comunicación se han considerado como importantes e independientes para ser adquiridas por los estudiantes; sin embargo, representan una parte fundamental de la consulta.

Objetivo. Describir un programa formativo de integración de habilidades de anamnesis y comunicación para estudiantes de tercero y analizar la correlación entre ambos dominios.

Sujetos y métodos. Estudio observacional descriptivo. Emplazamiento: Aula de Habilidades de la Facultad de Medicina de Córdoba. Muestra: 158 estudiantes de tercero de medicina. Intervenciones: se ha desarrollado un programa interactivo en habilidades de comunicación y realización de anamnesis; un evaluador independiente valoró el desarrollo de ambas durante la entrevista clínica de los encuentros videograbados con una paciente simulada utilizando una herramienta ad hoc.

Resultados. Duración media de cada entrevista: 5:56 min (IC 95\% = 5:38-6:15 min), con un máximo de 10 min. Puntuación media global de la escala de valoración: 56,2 (máx. 104) (IC 95\% = 54,20-58,19); habilidades de relación médicopaciente puntuación media: 25,58 (máx. 40) (IC 95\% = 24,7-26,5); anamnesis biomédica: 30,61 (máx. 64) (IC 95\% = 29,20-32,03). Existe una correlación positiva entre el tiempo usado y la puntuación global obtenida en la escala de valoración (coeficiente de Pearson $=0,65 ; p<0,01$ ) y entre los apartados biomédicos y comunicación (coeficiente de Pearson = $0,44 ; p<0,01)$.

Conclusiones. El programa fue factible; las habilidades en comunicación que mejor se llevaron a cabo fueron: reactividad, uso del lenguaje no verbal, recibimiento y cierre de entrevista. El tiempo es una variable importante para desarrollar las habilidades aprendidas y la mayoría de los estudiantes no agotan el disponible. Los estudiantes que mejor abordan los aspectos relacionales también consiguen más información en la anamnesis.

Palabras clave. Anamnesis. Estudiantes. Habilidades en comunicación.

\section{A program to help third year students to integrate content and form in the clinical history}

Introduction. Communication skills are considered as important and independent capacity to be acquired by students, however, are a fundamental part of the consultation.

Aim. To describe a formative program for third year students with the objective of integrating anamnesis and communication skills in an integrating way and evaluate the correlation between them both.

Subjects and methods. Observational descriptive study. Location: Skills Classroom in the Medicine Faculty of Cordoba. Subjects: 158 3rd year medicine students. Interventions: an interactive program in communications skills in clinical stories performances has been developed. An independent evaluator valued the communication and anamnesis skills during the clinical interview of the recorded meetings with simulated patient using a tool ad hoc.

Results. Average interview time: 5:56 $\mathrm{min}(95 \% \mathrm{Cl}=5: 38-6: 15 \mathrm{~min}$ ) (maximum $10 \mathrm{~min}$ ). Average global score rating scale: 56.2 (maximum 104) $(95 \% \mathrm{Cl}=54.20-58.19)$; skills of doctor-patient average score: 25.58 (maximum 40$)(95 \% \mathrm{Cl}=$ 24.7-26.5), and biomedical anamnesis: 30.61 (maximum 64) (95\% Cl $=29.20-32.03)$. A positive correlation exists between with the time used by students and the obtained punctuation in the rating scale (Pearson coefficient $=0.65 ; p<$ 0.01), just like in the biomedical and communication sections (Pearson coefficient $=0.44 ; p<0.01$ ).

Conclusions. The program was feasible, in which the communications skills had the best performance: reactivity, nonverbal language, reception and closing interview; against a not well performed biomedical anamnesis. Time is an important variable to develop the skills learnt and the students uses less than the maximum. The group of students with the best psychosocial aspects, got more details of anamnesis in their interviews.

Key words. Anamnesis. Communication skills. Students.

Unidad Docente de Medicina Familiar y Comunitaria de Córdoba. Córdoba, España.

Correspondencia: Dra. Isabel Salido Morales. Unidad Docente de Medicina Familiar y Comunitaria de Córdoba. Blanco Soler, 2- E-14004 Córdoba.

E-mail:

isa1111984@hotmail.com

Financiación:

Sociedad Andaluza de Medicina de Familia y Comunitaria (SAMFYC), Becas Isabel Fernández (expte. 89/1) Universidad de Córdoba, 'Proyectos de mejora de la calidad docente', curso 2009-2010 (expte. 093007).

Conflicto de intereses: No declarado.

Competing interests: None declared.

(c) 2012 Educación Médica 


\section{Introducción}

Las habilidades en comunicación y ciertas actitudes y valores que los profesionales deben mantener hacia los pacientes son competencias clínicas integradas con otras como el conocimiento, las habilidades para solucionar problemas y tomar decisiones, y las habilidades para usar recursos y de exploración física. Su uso conjunto va a favorecer un desarrollo de la consulta más eficiente $[1,2]$.

Hasta el momento, esta capacidad de comunicación se ha considerado importante y necesaria para una adecuada relación clínica y el buen funcionamiento de la consulta, y debe ser inherente al profesional sanitario que ejerce activamente [3]. El uso efectivo de estas habilidades por los médicos en el ámbito clínico beneficia tanto al profesional como a sus pacientes, comprobándose la asociación positiva de resultados de la consulta como satisfacción, adhesión y resultados de salud [4,5]. Desde esta perspectiva se resalta la importancia en la entrevista de aspectos como: abarcar sentimientos, considerar el impacto en la vida del paciente y su contexto biopsicosocial, en lugar de estar únicamente limitada a la perspectiva biológica [6,7].

La forma de comunicación que el médico tiene con sus pacientes es a través de la entrevista clínica. Existe divergencia de caracteres en cuanto al tipo de historia clínica a realizar: el modelo tradicional se centra en los procesos patológicos, en detrimento de la comprensión del paciente, de sus necesidades y las emociones o pensamientos que sienten sobre su enfermedad, que es el modelo de historia clínica denominado 'centrada en el paciente' [8]. Hasta ahora, ambas competencias eran impartidas por separado y no de forma integral, por lo que en el ámbito de la educación médica parece existir una dualidad entre la forma y el contenido de la entrevista médica.

La incorporación de la comunicación en los planes docentes de grado se contempla como un requisito importante en los planes de estudios, como una competencia que los estudiantes deben adquirir -aprobada en el año 2008 [9]-, y hacerlo de manera integrada con la anamnesis es una estrategia educativa clave. En este sentido se han hecho propuestas como el uso de las guías Calgary-Cambridge [6] o las que utilizan métodos educativos interactivos donde el alumno puede enfrentarse a situaciones de bajo riesgo y poner en práctica sus habilidades comunicativas [10-12]. En España existe poca experiencia en el uso de este tipo de formación a pesar de que estos encuentros permiten a los estudiantes hacer, reconocer y corregir errores, espe- cialmente si la interacción va seguida de un feedback docente (que resalte puntos fuertes y fomente la confianza) y de una autorreflexión que facilite la continuación de su proceso de autoaprendizaje en habilidades de comunicación $[10,13,14]$. Con estos tipos de métodos se ha podido comprobar que la forma de comportarse de los profesionales puede transformarse y mejorarse, y que estas modificaciones pueden perdurar a lo largo de los años [2].

De esta forma, en este estudio nos planteamos los siguientes objetivos:

- Desarrollar un programa práctico para que los estudiantes integren las habilidades comunicativas básicas con las de obtención de información al realizar una historia clínica.

- Describir en qué medida llevan esto a la práctica: qué habilidades y conductas comunicativas y de anamnesis se emplean con mayor y menor frecuencia.

- Conocer si el nivel de comunicación que utiliza el estudiante se relaciona con la calidad de la información que obtiene en la historia.

\section{Sujetos y métodos}

Se ha diseñado un programa docente, tanto teórico como práctico, sobre formación en entrevista clínica y habilidades en comunicación a los estudiantes de tercero de medicina. A partir de su programa práctico, se ha planteado un estudio observacional descriptivo para analizar cómo llevan a cabo los estudiantes una entrevista clínica con una paciente simulada.

En el estudio han participado 158 alumnos de tercer curso de medicina no rematriculados en la asignatura de 'Patología General'. Se ha llevado a cabo en el contexto del programa de prácticas de esta asignatura en el Aula de Habilidades de la Facultad de Medicina de Córdoba. El programa ha sido impartido por profesores asociados a la asignatura, procedentes de atención primaria.

\section{Esquema de desarrollo del estudio}

En el programa docente de formación de pregrado de 'Patología General' (asignatura de tercer curso), la sección de actividades prácticas se compone de seis actividades. En la primera, los estudiantes han aprendido a realizar una historia clínica centrada en la persona, teniendo en cuenta el problema biomédico y también su perspectiva biopsicosocial. Esta práctica se desarrolló en dos partes. Una primera teórica/práctica, donde se mostraba a los alum- 
nos cómo realizar una historia clínica completa y se les daba la oportunidad de practicarla. Para ello se usó como metodología:

- Una clase teórica sobre los principios básicos para realizar una historia clínica centrada en la persona, tanto en aspectos de contenido (anamnesis tradicional) como de forma (habilidades de comunicación).

- Visualización en video de un encuentro médicopaciente, donde se ejemplificaban las características fundamentales de una entrevista clínica con un paciente, así como las tareas de anamnesis.

- Role playing entre alumno y jefe de prácticas.

- Discusión individual o en grupo con feedback docente sobre la actuación del alumno.

En la segunda parte, cada alumno tuvo la oportunidad de entrevistar a una paciente simulada y recibir feedback sobre su actuación videograbada.

El encuentro simulado tuvo una duración límite de 10 minutos, se realizó en una consulta simulada en el laboratorio de habilidades y se grabó en video. Tras su realización, el alumno y la paciente simulada vieron conjuntamente la videograbación y esta última dio un feedback constructivo al alumno.

La paciente simulada recibió un detallado entrenamiento previo, por parte de un experto en comunicación clínica, sobre la historia clínica que debía representar y sobre cómo ofrecer un feedback estructurado posterior a la entrevista a los estudiantes. Se elaboró un guión donde se explicaban con detalle todos estos aspectos. El caso clínico interpretado fue el mismo para todos los alumnos (Tabla I).

Para completar el método docente de formación, los estudiantes realizaron un informe de autorreflexión sobre el encuentro simulado en su cuaderno de prácticas. El libro de prácticas-portafolio utilizado por los alumnos se diseñó para que los alumnos reflexionasen sobre todas las actividades de las prácticas y, también, para documentar las actividades realizadas y su dominio. Se usa como método docente y de aprendizaje de competencias formativas en residentes de medicina y se ha comenzado a implantar en la Facultad de Córdoba con los estudiantes de tercer año en este programa de formación en habilidades en comunicación.

Para llevar a cabo el análisis de las habilidades comunicativas y de obtención de información, durante la entrevista se usó una escala de valoración adaptada a partir de la escala CICAA $[15,16]$, a la que se incorporaron las tareas y habilidades de la anamnesis.

El conjunto de esta nueva escala fue de 52 ítems. Se seleccionaron de la CICAA original las tres primeras tareas relacionadas con habilidades comuni-
Tabla I. Caso clínico.

Mujer de 42 años de edad, que presenta cefalea holocraneal descrita como una presión de unos tres meses de evolución, que comienza por la mañana y empeora progresivamente a lo largo del día. Se acompaña de sensación nauseosa y estreñimiento.

Ella es auxiliar de clínica y se ha automedicado (paracetamol + ibuprofeno + codeína), pero con escasa mejoría clínica.

Antecedentes personales: mujer sana previamente, divorciada y casada por segunda vez, y actualmente en trámites de adopción por problemas de fertilidad de ella.

Antecedentes familiares: su padre falleció hace 24 años de accidente de tráfico y su madre hacía tres meses de tromboembolismo pulmonar. Su madre vivía con ella y tenían una estrecha relación. Su hermano vive en México y no mantienen apenas contacto.

Tabla II. Concordancia intraobservador para la escala de valoración de entrevista clínica

\begin{tabular}{lcl}
\hline Índice kappa y grado de acuerdo & $\%$ & Ítems valorados \\
\hline Pobre $(<0,20)$ & 1,9 & 3 \\
\hline Débil $(0,21-0,40)$ & 7,7 & $5,18,25,46$ \\
\hline Moderado $(0,41-0,60)$ & 13,5 & $2,6,9,15,26,27,52$ \\
\hline Bueno $(0,61-0,80)$ & 26,9 & $4,8,11,17,20,22,28,31,33,37,41,49,50$ \\
\hline Muy bueno $(>0,81)$ & 50,0 & $\begin{array}{l}1,7,10,12,13,14,16,19,21,23,24,29,30,32, \\
34,35,36,38,39,40,42,43,44,45,47,48,51\end{array}$ \\
\hline
\end{tabular}

cativas y estrechamente ligadas a la relación médico-paciente: conectar, identificar y comprender el o los problemas de salud de la paciente. Corresponden los primeros 20 ítems. Los demás 32 ítems corresponden a las habilidades en realización de anamnesis desde el punto de vista médico: identificar el problema de salud (síntomas que se presentan, tanto el principal como los secundarios, revisión por aparatos y sistemas), antecedentes personales, familiares, y contexto social y repercusión que este problema de salud puede tener en la vida de los pacientes. Cada ítem se valora de 0 a 2 puntos ( 0 : conducta/habilidad ausente; 1 : probablemente presente; 2: presente).

Un evaluador externo revisó las entrevistas clínicas y realizó su análisis. Previamente llevó a cabo un entrenamiento con expertos observadores, diestros en entrevista clínica, con el fin de aprender y manejar la valoración de habilidades en entrevista con residentes, médicos, enfermeros y estudiantes. La fiabilidad intraobservador se valoró utilizando un test-retest con 25 entrevistas. Los ítems de escala de valoración de entrevista se analizaron mediante el índice kappa (Tabla II), y el índice de correla- 
Tabla III. Resultados obtenidos por los estudiantes en los distintos ítems de la escala de valoración de habilidades.

\begin{tabular}{|c|c|c|c|}
\hline & 0 puntos & 1 punto & 2 puntos \\
\hline \multicolumn{4}{|l|}{ Conectar con el paciente/familia } \\
\hline ¿Recibe adecuadamente al paciente? & $0(0,0 \%)$ & $10(6,3 \%)$ & $148(93,7 \%)$ \\
\hline $\begin{array}{l}\text { ¿Hace uso del ordenador u otros registros } \\
\text { de forma que no altera la comunicación? }\end{array}$ & $112(70,9 \%)$ & $24(15,2 \%)$ & $22(13,9 \%)$ \\
\hline ¿Se muestra cortés y amable durante la entrevista? & $0(0,0 \%)$ & $13(8,2 \%)$ & $145(9,8 \%)$ \\
\hline ¿Su lenguaje no verbal es el adecuado? & $0(0,0 \%)$ & $20(12,7 \%)$ & $138(87,3 \%)$ \\
\hline ¿Muestra empatía en los momentos oportunos? & $18(11,4 \%)$ & $54(34,2 \%)$ & $86(54,4 \%)$ \\
\hline ¿Cierra adecuadamente la entrevista con el paciente? & $5(3,2 \%)$ & $52(32,9 \%)$ & $101(63,9 \%)$ \\
\hline \multicolumn{4}{|l|}{ Identificar el problema: perspectiva del paciente } \\
\hline ¿Ha mostrado una reactividad adecuada? & $3(1,9 \%)$ & $28(17,7 \%)$ & $127(80,4 \%)$ \\
\hline ¿Facilita el discurso del paciente? & $4(2,5 \%)$ & $31(19,6 \%)$ & $123(77,8 \%)$ \\
\hline $\begin{array}{l}\text { ¿Establece y mantiene a lo largo de la entrevista } \\
\text { un contacto visual-facial adecuado? }\end{array}$ & $0(0,0 \%)$ & $12(7,6 \%)$ & $146(92,4 \%)$ \\
\hline ¿Capta y responde las pistas ofrecidas por el paciente? & $70(44,3 \%)$ & $43(27,2 \%)$ & $45(28,5 \%)$ \\
\hline ¿Emplea preguntas abiertas? & $52(32,9 \%)$ & $64(40,5 \%)$ & $42(26,6 \%)$ \\
\hline $\begin{array}{l}\text { ¿Ha explorado la idea que tenía el propio paciente } \\
\text { sobre el origen o causa de su síntoma o proceso? }\end{array}$ & $35(22,2 \%)$ & $19(12,0 \%)$ & $104(65,8 \%)$ \\
\hline $\begin{array}{l}\text { ¿Ha explorado las emociones y sentimientos } \\
\text { que el síntoma o proceso ha provocado al paciente? }\end{array}$ & $99(62,7 \%)$ & $34(21,5 \%)$ & $25(15,8 \%)$ \\
\hline $\begin{array}{l}\text { ¿Ha explorado como afecta al paciente su síntoma } \\
\text { o proceso en su vida diaria, entorno sociofamiliar o laboral? }\end{array}$ & $68(43,0 \%)$ & $19(12,0 \%)$ & $71(44,9 \%)$ \\
\hline ¿Ha explorado las expectativas que el paciente tiene para esta consulta? & $115(72,8 \%)$ & $34(21,5 \%)$ & $9(5,7 \%)$ \\
\hline ¿Ha explorado el estado de ánimo del paciente? & $73(35,4 \%)$ & $22(13,9 \%)$ & $63(39,9 \%)$ \\
\hline ¿Ha explorado posibles acontecimientos vitales estresantes para el paciente? & $56(35,4 \%)$ & $31(19,6 \%)$ & $71(44,9 \%)$ \\
\hline ¿Ha explorado el entorno sociofamiliar? & $52(32,9 \%)$ & $9(5,7 \%)$ & $97(61,4 \%)$ \\
\hline $\begin{array}{l}\text { ¿Ha explorado si el paciente ha tomado alguna medida } \\
\text { para solucionar/afrontar el problema? }\end{array}$ & $15(9,5 \%)$ & $4(2,5 \%)$ & $139(88 \%)$ \\
\hline ¿Ha resumido la información que ha obtenido del paciente? & $95(60,1 \%)$ & $18(11,4 \%)$ & $45(28,5 \%)$ \\
\hline \multicolumn{4}{|l|}{ Identificar el problema: perspectiva biomédica. Síntomas principales } \\
\hline ¿En qué medida el alumno ha explorado la naturaleza o tipo de síntoma? & $1(0,6 \%)$ & $54(34,2 \%)$ & $103(65,2 \%)$ \\
\hline ¿La forma de inicio? & $27(17,1 \%)$ & $44(27,8 \%)$ & $87(55,1 \%)$ \\
\hline ¿La localización? & $40(25,3 \%)$ & $12(7,6 \%)$ & $106(67,1 \%)$ \\
\hline ¿La irradiación? & $43(27,2 \%)$ & $20(7,6 \%)$ & $95(60,1 \%)$ \\
\hline ¿La evolución? & $0(1,3 \%)$ & $8(5,1 \%)$ & $148(93,6 \%)$ \\
\hline ¿Los factores que lo modifican? & $13(8,2 \%)$ & $68(43,0 \%)$ & $77(48,7 \%)$ \\
\hline ¿Otros síntomas asociados? & $19(12,0 \%)$ & $42(26,6 \%)$ & $97(61,4 \%)$ \\
\hline
\end{tabular}

ción intraclase (ICI), para la puntuación total de la escala $(0,948$; IC $95 \%=0,89-0,98)$ y para los apartados de relación $(0,9$; IC $95 \%=0,79-0,96)$ y de perspectiva biomédica $(0,961$; IC $95 \%=0,91-0,98)$.
Se llevó a cabo un análisis estadístico descriptivo e inferencial, aplicando el coeficiente de correlación de Pearson para comprobar la correlación entre la puntuación obtenida con la escala de valoración de 
Tabla III. Resultados obtenidos por los estudiantes en los distintos ítems de la escala de valoración de habilidades (cont.).

\begin{tabular}{|c|c|c|c|}
\hline & 0 puntos & 1 punto & 2 puntos \\
\hline \multicolumn{4}{|l|}{ Identificar el problema: perspectiva biomédica. Revisión por órganos y aparatos } \\
\hline Aparato respiratorio & $54(34,2 \%)$ & $51(32,3 \%)$ & $53(33,5 \%)$ \\
\hline Sistema cardiovascular & $77(48,7 \%)$ & $40(25,3 \%)$ & $41(25,9 \%)$ \\
\hline Sistema nervioso & $79(50,0 \%)$ & $25(15,8 \%)$ & $54(34,2 \%)$ \\
\hline Aparato digestivo & $29(18,4 \%)$ & $29(18,4 \%)$ & $100(63,3 \%)$ \\
\hline Sistema genitourinario & $107(67,7 \%)$ & $12(7,6 \%)$ & $39(24,7 \%)$ \\
\hline Musculoesquelético & $98(62,0 \%)$ & $18(11,4 \%)$ & $42(26,6 \%)$ \\
\hline Endocrinológico & $143(90,5 \%)$ & $6(3,8 \%)$ & $9(5,7 \%)$ \\
\hline Piel & $153(96,8 \%)$ & $1(0,6 \%)$ & $4(2,5 \%)$ \\
\hline Sistema cognitivo & $137(86,7 \%)$ & $7(4,4 \%)$ & $14(8,9 \%)$ \\
\hline Psiquiátrico & $83(52,5 \%)$ & $23(14,6 \%)$ & $52(32,9 \%)$ \\
\hline \multicolumn{4}{|l|}{ Identificar el problema: perspectiva biomédica. Antecedentes médicos personales } \\
\hline Antecedentes de enfermedades previas & $38(24,1 \%)$ & $16(10,1 \%)$ & $104(65,8 \%)$ \\
\hline Ingresos hospitalarios/operaciones & $81(51,3 \%)$ & $2(1,3 \%)$ & $75(47,5 \%)$ \\
\hline Accidentes y heridas & $126(79,7 \%)$ & $3(1,9 \%)$ & $29(18,4 \%)$ \\
\hline Consumo de fármacos & $16(10,1 \%)$ & $76(48,1 \%)$ & $66(41,8 \%)$ \\
\hline Antecedentes ginecológicos: embarazos/abortos & $63(39,9 \%)$ & $4(2,5 \%)$ & $91(57,6 \%)$ \\
\hline \multicolumn{4}{|l|}{ Información contextual: perfil del paciente } \\
\hline Historia ocupacional & $58(36,7 \%)$ & $14(8,9 \%)$ & $86(54,4 \%)$ \\
\hline Estilo de vida: hábitos dietéticos & $103(65,2 \%)$ & $7(4,4 \%)$ & $48(30.4 \%)$ \\
\hline Estilo de vida: consumo de alcohol & $75(47,5 \%)$ & $1(0,6 \%)$ & $82(51,9 \%)$ \\
\hline Estilo de vida: consumo de tabaco & $69(43,7 \%)$ & $1(0,6 \%)$ & $88(55,7 \%)$ \\
\hline Estilo de vida: consumo de otras drogas & $139(88,0 \%)$ & $0(0,0 \%)$ & $19(12,0 \%)$ \\
\hline Estilo de vida: práctica de ejercicio & $113(71,5 \%)$ & $3(1,9 \%)$ & $42(26,6 \%)$ \\
\hline Viajes & $141(89,2 \%)$ & $0(0,0 \%)$ & $17(10,8 \%)$ \\
\hline Historia familiar: posibles alteraciones hereditarias & $37(23,4 \%)$ & $20(12,7 \%)$ & $101(63,9 \%)$ \\
\hline Historia familiar: enfermedades y causas de muerte en otros miembros & $39(24,7 \%)$ & $33(20,9 \%)$ & $86(54,4 \%)$ \\
\hline $\begin{array}{l}\text { Historia familiar: patrones de comportamiento, } \\
\text { relaciones intrafamiliares, fuentes de apoyo/estrés... }\end{array}$ & $87(55,1 \%)$ & $46(29,1 \%)$ & $25(15,8 \%)$ \\
\hline
\end{tabular}

la entrevista y el tiempo empleado en la entrevista clínica ( $p<0,05$; contrastes bilaterales). Se usó el programa estadístico SPSS v. 17.0 para Windows.

\section{Resultados}

Se analizaron un total de 158 videograbaciones mediante una escala de valoración de entrevista clínica, obteniéndose una media de 56,2 $\pm 12,6$ puntos, con respecto a 104 del total posible (IC 95\% = 54,21$58,19)$. La máxima puntuación obtenida en el cuestionario fue de 92, y la mínima, de 22. La duración media de la entrevista fue de 5 minutos y 56 segundos (IC 95\% = 5:38-6:15 min), con una duración máxima de 10 minutos.

En la tabla III se recogen los resultados de los ítems analizados. Cabe señalar que se ha obtenido una media de 9,16 puntos (de un máximo de 12) en las habilidades comunicativas mostradas por los es- 
Tabla IV. Correlación entre escala de valoración en entrevista y tiempo.

\begin{tabular}{|c|c|c|c|c|c|}
\hline & & Tiempo & $\begin{array}{l}\text { Puntuación total en la } \\
\text { escala de valoración }\end{array}$ & $\begin{array}{l}\text { Escala de valoración } \\
\text { (relación/personal) }\end{array}$ & $\begin{array}{l}\text { Escala de valoración } \\
\text { (biomédico) }\end{array}$ \\
\hline Tiempo & Correlación de Pearson & 1 & $0,647^{a}$ & $0,493^{a}$ & $0,570^{a}$ \\
\hline \multirow{2}{*}{$\begin{array}{l}\text { Puntuación total en } \\
\text { la escala de valoración }\end{array}$} & Significación (bilateral) & & 0,000 & 0,000 & 0,000 \\
\hline & Correlación de Pearson & $0,647^{a}$ & 1 & $0,769^{a}$ & $0,914^{a}$ \\
\hline \multirow{2}{*}{$\begin{array}{l}\text { Escala de valoración } \\
\text { (relación/personal) }\end{array}$} & Significación (bilateral) & 0,000 & & 0,000 & 0,000 \\
\hline & Correlación de Pearson & $0,493^{a}$ & $0,769^{a}$ & 1 & $0,444^{a}$ \\
\hline \multirow{2}{*}{$\begin{array}{l}\text { Escala de valoración } \\
\text { (biomédico) }\end{array}$} & Significación (bilateral) & 0,000 & 0,000 & & 0,000 \\
\hline & Correlación de Pearson & $0,570^{a}$ & $0,914^{a}$ & $0,444^{a}$ & 1 \\
\hline
\end{tabular}

tudiantes para conectar con la paciente simulada. Las habilidades mejor realizadas en este apartado las efectuaron el 93,7\% de los estudiantes con un recibimiento correcto de la paciente, y el 91,8\% que se mostraron corteses y amables a lo largo de la entrevista.

La puntuación media en las habilidades mostradas para identificación del problema de salud fue de 16,36 sobre 28. Las habilidades de este apartado que mejor realizaron los estudiantes fueron: mantener una reactividad adecuada $(80,4 \%)$, facilitar el discurso de la paciente $(77,8 \%)$ y establecer contacto visual $(92,4 \%)$. La mayoría de los estudiantes no tuvieron en cuenta las emociones o sentimientos que el síntoma estaba produciendo en la paciente $(62,7 \%)$, sus expectativas para la consulta $(72,8 \%)$, ni captar las pistas que ofrecía la paciente a lo largo de la entrevista $(44,3 \%)$.

En general, los estudiantes mostraron habilidades comunicativas para identificar el problema de salud desde una perspectiva biomédica, con 10,61 como puntuación media (máximo de 14). Lo más explorado fue la evolución del síntoma guía $(93,6 \%$ de los estudiantes) y los factores que lo modificaban $(91,7 \%)$. En cambio, se obtuvo una media de 6 puntos (sobre 20) en habilidades comunicativas de anamnesis de diferentes aparatos y sistemas, con un porcentaje más alto en anamnesis sobre síntomas que padece la paciente en el sistema digestivo y muy bajo en el sistema endocrino o musculoesquelético.

En la revisión de anamnesis para obtención de información sobre antecedentes personales de la paciente y su contexto sociofamiliar se obtuvo una puntuación media de 13,59 sobre un total de 30, con una puntuación máxima de 28 y mínima de 2 . Un $75,9 \%$ de los estudiantes preguntaron a la paciente sobre sus antecedentes médicos y un $89,9 \%$ tuvieron en cuenta si la paciente podía estar siguiendo algún tipo de tratamiento. Un 76,6 \% de los estudiantes realizaron anamnesis dirigida a una posible relación entre el síntoma de la paciente y la posibilidad de existencia de un patrón familiar, y un $75,3 \%$ preguntaron sobre posibles enfermedades o causas de muerte de los familiares. Muy pocos tuvieron en cuenta el consumo de tabaco y alcohol (55,7\% y $51 \%$, respectivamente), y aún menos el consumo de drogas (12\%).

Existía una correlación positiva entre el tiempo empleado por los estudiantes para llevar a cabo la entrevista clínica y la puntuación que obtenida en la escala de valoración usada (coeficiente de correlación de Pearson $=0,65 ; p<0,01$ ). De la misma forma, los estudiantes que mejor realizaron la entrevista clínica en el aspecto biomédico se centraron en los aspectos relacionales y personales de la paciente, con una correlación positiva (coeficiente de Pearson $=0,44 ; p<0,01)$ y con el tiempo empleado (coeficiente de Pearson $=0,5 ; p<0,01$ para puntuación del apartado biomédico; coeficiente de Pearson $=0,57 ; p<0,01$ para apartado comunicacional). En las tablas IV y V se muestran los resultados obtenidos y, además, un análisis de las entrevistas dividiendo los resultados en cuartiles para apreciar la correlación de las mejores puntuaciones obtenidas en ambas escalas. 


\section{Discusión}

La enseñanza de habilidades en relación clínica propuestas en este programa suponen un sistema integral de técnicas docentes que se pueden resumir en: formación teórica en habilidades de comunicación, práctica específica de las mismas, observación con feedback y repetición de las habilidades aprendidas, y realización de una entrevista clínica simulada y un portafolio reflexivo posterior.

En este sentido, este programa ensayado ha resultado ser factible dentro del apartado práctico de la asignatura de 'Patología General' en tercer curso de medicina, y sigue la línea de otros programas que intentan integrar las habilidades de comunicación y anamnesis y que han probado su efectividad $[13,14,17]$. Existe más experiencia del uso de estos métodos con residentes de medicina, pero ahora se están comenzando también a realizar con estudiantes de otras áreas sanitarias, como enfermería u odontología $[11,12,18]$.

En relación a los resultados, la mayoría de los estudiantes obtuvieron un nivel de tareas realizadas aceptable, especialmente si consideramos que era la primera vez o de las primeras experiencias que tenían con la práctica médica clínica. Probablemente, las actividades formativas sobre cómo realizar una entrevista clínica y todo el entrenamiento previo durante las prácticas ayudaron a los estudiantes a saber cómo afrontar este primer contacto en el papel de médico con una paciente simulada.

Los estudiantes obtienen mejor puntuación porcentual si comparamos las habilidades comunicativas con las de anamnesis del problema biomédico. De las primeras, las más destacables son sobre todo las mostradas para conectar con la paciente: establecieron un recibimiento correcto, se mostraron educados, con empatía sobre su problema, y usaron un lenguaje no verbal adecuado. También identificaron el problema de salud desde el punto de vista más personal: mantuvieron un contacto visual estrecho, mostraron reactividad correcta y facilitaron el discurso de la paciente. Estos resultados son similares a los obtenidos en otros programas, como el de entrenamiento en habilidades de comunicación con estudiantes de odontología y medicina en la Universidad de Virginia [11], donde las habilidades que mejor aprendieron coinciden con las habilidades que mejor han realizado los estudiantes de Córdoba. Este hecho nos hace considerar cuáles eran sus capacidades previas en comunicación para llevar a cabo la entrevista, ya que en el ámbito de las conductas comunicativas es posible que muchas de estas habilidades sean inhe-
Tabla V. Porcentaje de puntuación de escala de valoración en habilidades en comunicación y anamnesis biomédica, dividida en cuartiles.

\begin{tabular}{cccccc}
\hline & 1,00 & 2,00 & 3,00 & 4,00 & Total \\
\hline 1,00 & $20(12,7 \%)$ & $10(6,4 \%)$ & $4(2,5 \%)$ & $6(3,8 \%)$ & $40(25,5 \%)$ \\
\hline 2,00 & $6(3,8 \%)$ & $9(5,7 \%)$ & $13(8,3 \%)$ & $9(5,7 \%)$ & $37(23,6 \%)$ \\
\hline 3,00 & $10(6,4 \%)$ & $7(4,5 \%)$ & $12(7,6 \%)$ & $11(7,0 \%)$ & $40(25,5 \%)$ \\
\hline 4,00 & $2(1,3 \%)$ & $10(6,4 \%)$ & $9(5,7 \%)$ & $19(12,1 \%)$ & $40(25,5 \%)$ \\
\hline Total & $38(24,2 \%)$ & $36(22,9 \%)$ & $38(24,2 \%)$ & $45(28,7 \%)$ & $157(100 \%)$ \\
\hline
\end{tabular}

rentes a la personalidad. Otras habilidades, como captar pistas, indagar en los sentimientos que el problema de salud crea en la paciente o explorar las expectativas de la paciente para la consulta, han sido muy poco desarrolladas por los estudiantes a pesar de haber tenido un entrenamiento previo, probablemente porque son habilidades más específicas y que requieren un aprendizaje más intenso y mayor experiencia en la realización de entrevistas clínicas repetidas.

Aunque no era el objetivo de este estudio, hubiera sido de utilidad conocer el grado de habilidades y capacidades comunicativas previas de los estudiantes, ya que ello habría permitido valorar mejor el alcance del programa ensayado y la posterior evolución de las habilidades comunicacionales estudiadas en comparación con las de tipo biomédico.

Es de destacar que, a pesar de tener un tiempo límite de 10 minutos, los estudiantes usen poco más de la mitad, y que sobrándoles tiempo de entrevista, exploren pobremente el problema biomédico, la anamnesis por aparatos y sistemas, y los antecedentes personales y familiares de la paciente. Se podría pensar que los estudiantes se muestran nerviosos ante el hecho de ser la primera vez que se les grababa en una entrevista clínica. La mayoría de nuestros estudiantes, una vez extraídos algunos datos de anamnesis, no continúan profundizando más en ella, no sabemos muy bien si por intimidación de la cámara y su deseo de terminar lo antes posible la entrevista, o bien por falta de conocimiento sobre qué y cómo indagar en la anamnesis.

El hecho de usar poco tiempo en la entrevista con los estudiantes ya se había constatado en el proyecto COMCORD [12], donde se comprobó una relación directamente proporcional entre el tiempo empleado en la consulta y las habilidades de comunicación mostradas por los residentes, así como la 
reducción del tiempo de entrevista a medida que el residente iba completando su formación. Pero aunque los resultados sean superponibles, existen diferencias entre ambos. Es muy probable que los estudiantes usen menos tiempo de entrevista debido a la falta de formación teórica sobre exploración médica, mientras que los residentes, tras una formación casi exclusivamente biomédica, se centran más en este contenido médico del problema.

Otro hallazgo de gran interés es la constatación de que los estudiantes que mejores habilidades en comunicación despliegan son aquellos que mejor puntuación obtienen en tareas de anamnesis del problema biomédico, y, como se ha comentado, son los que mayor tiempo de entrevista consumen. En los resultados de diferentes investigaciones es un hecho que tanto los residentes más jóvenes como los estudiantes hacen mejor las habilidades de comunicación que los que están a punto de finalizar su formación, y también parece que son más sensibles a estos temas al observarse en ellos una correlación negativa entre su actuación clínica y la edad $[12,18]$. Tras mejorar su aprendizaje en temas clínicos, empeoran sus habilidades comunicativas. Pero en la práctica médica, el uso de buenas habilidades de comunicación se ha relacionado con una mejora en la gestión del tiempo de consulta; así, los médicos que han desarrollado aspectos como la empatía, la exploración de creencias y expectativas de los pacientes, o la participación del paciente en las decisiones, finalmente llegan a consumir el mismo tiempo de consulta que los que no lo hacen [19].

Hoy día no se discute que debe darse formación posgraduada en habilidades de comunicación de entrevista a los residentes, pero es clave y más adecuado hacerlo en programas estructurados desde los primeros años del grado [12,20,21]. El programa de formación en habilidades de comunicación y anamnesis implementado en la Facultad de Medicina de Córdoba se revela factible y probablemente de utilidad para llevar un aprendizaje en habilidades de comunicación integrado con las de anamnesis tradicional y ponerlas en práctica. Sus resultados representan un buen material para su posterior análisis con el objetivo de valorar la evolución a lo largo de los años de carrera de las habilidades aprendidas por estos estudiantes.

En conclusión:

- El programa docente teórico-práctico implementado es de utilidad para que los estudiantes muestren sus habilidades de comunicación y de anamnesis biomédica, estableciendo discriminación entre ellos.
- El tiempo es una variable relacionada proporcionalmente con el desarrollo de las habilidades comunicacionales y de anamnesis.

- Las habilidades que los estudiantes mejor han llevado a cabo han sido conectar con el paciente e identificar su problema de salud desde una perspectiva más personal.

- Los estudiantes que mejor realizan la entrevista desde el punto vista comunicacional son también los que obtienen una información de más calidad en la anamnesis, y viceversa.

\section{Bibliografía}

1. Stevens A, Hernández J. The use of virtual patients to teach medical students history taking and communication skills. Am J Surg 2006; 191: 806-11.

2. Ruiz-Moral R. Relación clínica. Guía para aprender, enseñar e investigar. Barcelona: SEMFYC; 2004.

3. Moore P, Gómez G, Kurtz S, Vargas A. Doctor patient communication: which skills are effective? Rev Med Chile 2010; 138: 1047-54.

4. Cebriá J, Palma C, Segura J, García R, Pérez J. El entrenamiento en habilidades de comunicación podría ser un factor preventivo del síndrome de burnout en médicos de familia. Revista de Psiquiatría de la Facultad de Medicina de Barcelona 2006; 33: 34-40.

5. Tamblyn R, Abrahamowicz M, Dauphinee D, Wenghofer E, Jacques A, Klass D, et al Physician scores on a national clinical skills examination as predictors of complaints to medical regulatory authorities. JAMA 2007; 298: 993-1001.

6. Kurtz S, Sivlerman J, Benson J, Draper J. Marrying content and process in clinical method teaching: enhancing the Calgary-Cambridge Guides. Acad Med 2003; 78: 802-9.

7. Shapiro S, Lancee W, Rhichards-Bentley C. Evaluation of a communication skills program for first-year medical students at the University of Toronto. BMC Med Educ 2009; 9: 11.

8. Borrell F. El modelo biospsicosocial en evolución. Med Clin (Barc) 2002; 119: 175-9.

9. ECI/332/2008. Boletín Oficial del Estado, n. ${ }^{\circ 40 ; ~ p . ~ 8351-5 . ~}$

10. Ryan CA, Walshe N, Gaffney R, Shanks A, Burgoyne L, Wiskin CM. Using standardized patients to assess communication skills in medical and nursing students. BMC Med Educ 2010; 10: 24 .

11. Lanning SK, Ranson SL, Willett RM. Communication skills instruction utilizing interdisciplinary peer teachers: program development and student perceptions. J Dent Educ 2008; 72 172-82.

12. Rodríguez-Salvador JJ, Ruiz-Moral R. Programa de formación en técnicas de entrevista clínica: el proyecto COMCORD. Aten Primaria 2001; 27: 503-10.

13. Morrow JB, Dobbie AE, Jenkins C, Long R, Mihalic A, Wagner J. First-year medical students can demonstrate EHR-specific communication skills: a control-group study. Fam Med 2009; 41: 28-33.

14. Valero M, Aramburu J, Baños JE, Sentí M, Pérez J. Introducción de un portafolio para fomentar competencias transversales de los estudiantes universitarios. Educ Med 2007; 10: 244-51.

15. Gavilán-Moral E, Ruiz-Moral R, Pérula de Torres LA, Parras-Rejano JM. Valoración de la relación clínica centrada en el paciente: análisis de las propiedades psicométricas de la escala CICAA. Aten Primaria 2010; 42: 162-8.

16. Ruiz-Moral R, Pérula de Torres LA. Validez y fiabilidad de un instrumento para evaluar la comunicación clínica en las consultas: el cuestionario CICAA. Aten Primaria 2006; 37: 320-4.

17. Smith R, Lyles J, Metter J, Soffelmayr B, Van Egern L, Marshall A, et al. The effectiveness of intensive training for 
residents in interviewing. A randomized, controlled study. Ann Intern Med 1998; 128: 118-26.

18. Ruiz-Moral R, Parras-Rejano JM, Alcalá-Partera JA, CastroMartín E, Pérula de Torres LA. Welcome and I'll see you again or hello and goodbye?: communicative behaviour of medical residents in the first and last moments of consultations. Aten Primaria 2005; 36: 537-41.
19. Bellón JA. Communication skills and the utilisation of primary care consultations. An Sis Sanit Navarra 2001; 24 (Suppl 2): 7-14

20. Kurtz S, Silverman J, Draper J. Teaching and learning communication skills in medicine. Abingdon, UK: Radcliffe Medical Press; 1998.

21. Kidd J, Patel V, Peile E. Clinical and communication skills. Need to be learnt side by side. BMJ 2005; 330: 374-5. 